\title{
A new design of nanocrystalline silicon optical devices based on 3-dimensional photonic crystal structures
}

\author{
Daihei Hippo, Hea-Jeong Chong, Yoshiyuki Kawata, Atsushi Tanaka, Yoshishige Tsuchiya, Hiroshi Mizuta, Shunri Oda \\ Quantum NanoElectronics Research Center and Department of Physical Electronics, Tokyo Institute of Technology and SORST-JST, \\ Ookayama, Meguro-ku, Tokyo,152-8552, JAPAN \\ d-hippo@neo.pe.titech.ac.jp \\ Kei Urakawa, Nobuyoshi Koshida \\ Graduate School of Engineering, Tokyo University of Agriculture and Technology, Naka-cho Koganei Tokyo, 184-8588, JAPAN
}

\begin{abstract}
We propose a new design of nanocrystalline silicon optical devices which are based on control of electromagnetic fields, electronic states, as well as the phonon dispersion of size-controlled silicon quantum dots.
\end{abstract}

\section{Introduction}

Since optical gain was first reported for nanocrystalline silicon in 2000 by Pavesi et al [1], operation of silicon-based lasers have continuously been studied. Stimulated emission was reported in 2004 for a nanostructured silicon pn junction diode using current injection [2], and a silicon Raman laser has been demonstrated with pulsed optical pumping [3]. A continuous wave silicon Raman laser have also been reported recently [4].

Silicon based laser operation is expected as a key technology in realizing opto-electronic integrated circuits which circumvent the problem of interconnect bottleneck in the state-of-the-art ULSI technology. However, in the present state, silicon based lasers operate only under very high excitation conditions [2-4], making it difficult to incorporate them into the CMOS circuits in which ultra low power consumptions are required.

In this paper, we propose a completely different approach to realize silicon based laser operation at submicron scale using 3-dimensional photonic crystal structures combined with nanocrystalline silicon quantum dots. First, the 3-dimensional photonic crystal structures are introduced to increase the stimulated emission probability caused by the standing wave at the photonic band edge, thereby resulting in a significant increase in the external quantum efficiency. Second, nanocrystalline silicon quantum dots are used for constructing an active layer which emits light in the visible band due to the quantum confinement effect. As for the excitation mechanism, current injection is absolutely required for any practical applications. However, there has so far been no report on optical gain from nanocrystalline silicon under current injection although high efficiency band-edge electrolumminescence has already been reported for silicon light emitting diodes. One of the reasons is that current injection into nanocrystalline silicon causes a significant decrease in the internal quantum efficiency due to the interfacial oxide layer. To circumvent this problem we propose a new high energy electron emission approach which is unique to a nanocrystalline silicon dot array structure [5].

\section{Design and Fabrication Process of Silicon 3-Dimensional Photonic Crystal Structures}

We have designed a 3-dimensional full photonic badgap structures in the visible-band which aims at enhancing the stimulated emission probability significantly. We exploit the group velocity anomaly at the photonic band edge centered around 800nm which corresponds to the luminescence band of nanocrystalline silicon. The designed structure is similar to the woodpile one, but the fabrication process is completely different.

First, two sets of patterning are conducted on the top surface with just a half period shifted across a straight boundary, as shown in Fig.1. In the second process, the (111) plane is formed along the straight boundary using anisotropic alkaline etching or by mechanical polishing. At this stage, the patterning on the (111) plane is performed. In the final process, the second directional etching is performed in the direction perpendicular to the first directional etching according to the pattering on the (111) plane. In this way we obtain the double directionally etched structure which is theoretically expected to generate a maximum photonic bandgap, as shown in Fig.2 (a).

We use electron beam lithography to pattern the surface, and then anodic oxidation is performed under a magnetic field to realize the highly directional etching and therefore extremely a high aspect ratio. The physical mechanism behind this etching technique is the control of the motion of holes which are responsible for initiating the electrochemical dissolution of silicon. The directional etchings are realized through the cyclotron motion of the holes under a magnetic field. 


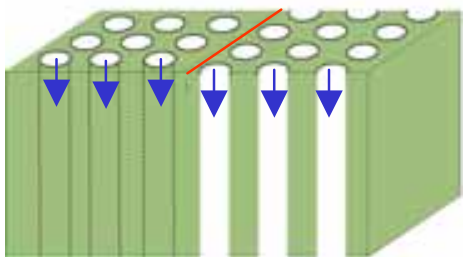

(a)

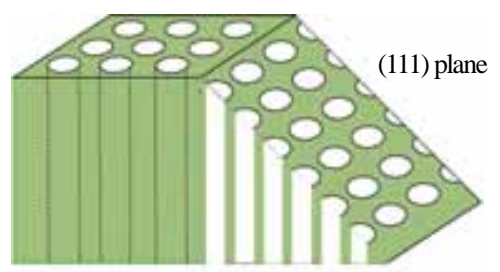

(b)

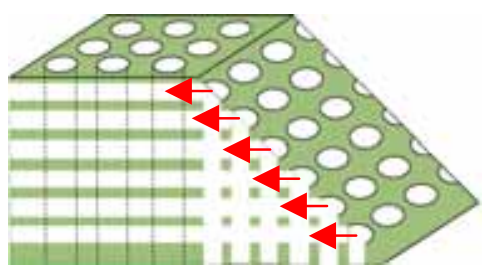

(c)

Fig.1. Fabrication processes of the 3-dimensional photonic crystal structure. (a) First directional etching, (b) anisotropic alkaline etching and (c) second directional etching.

The preliminary results of the first process are shown in Fig.2 (b). The straight vertical etching is realized using this method with the pore diameter of about $160 \mathrm{~nm}$. This dimension corresponds to the central wavelength of about $800 \mathrm{~nm}$ which is the typical luminescence wavelength of nanocrystalline silicon.

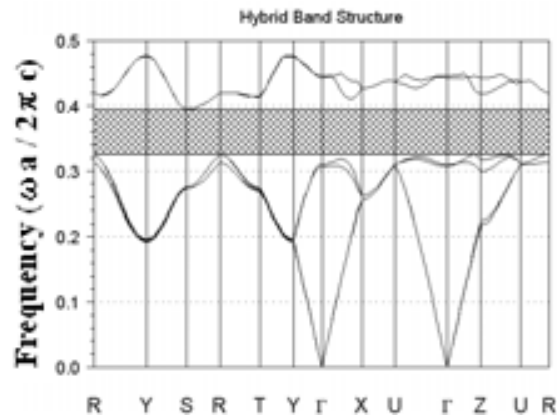

(a)

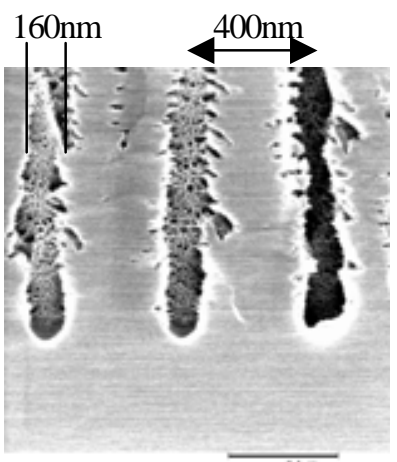

(b)

Fig.2. (a) Photonic band structure (b) Straight vertical etching with 160nm in diameter realized by anodic oxidation under magnetic field.

\section{Nanocrystalline Silicon Quantum Dots as an Emitter of Photons and Electrons}

Size-controlled nanocrystalline silicon quantum dots can be fabricated using a pulsed gas plasma CVD method [6], and the mean diameter is about $10 \mathrm{~nm}$, as shown in Fig.3. Note that each dot consists of single crystal silicon, and natural oxide is grown on its surface. The size of core silicon sphere can be controlled from 10 to $4 \mathrm{~nm}$ through thermal oxidation. Fig.4 shows the PL spectra observed for the oxidized samples, and a blue shift of the PL peaks is shown clearly associated with the quantum confinement effect in nanocrystalline silicon.

Next, we have examined the effect of introducing these nanocrystalline silicon dots into the pore formed on the top surface of the above mentioned photonic crystal structures. Assuming the refractive index of nanocrystalline silicon is the same as that for bulk silicon and the surrounding region basically consists of silicon dioxide considering the effect of thermal oxidation treatment, the photonic band gap varies according to the refractive index of the pores on the top surface, as shown in Fig.5. Suppose that core silicon quantum dot diameter is $10 \mathrm{~nm}$ and the oxide thickness is $2 \mathrm{~nm}$, the refractive index of pores will be about 1.83 (right dashed line in Fig.5). Note that the core diameter can be reduced by thermal oxidation and the refractive index of pores decreases accordingly. In addition, if the core diameter is reduced down to the quantum confinement regime, the silicon dots become transparent to the visible light due to the widening of the bandgap. So the refractive index of pores can be reduced to its minimum value which correspond to that of silicon dioxide (left dashed line in Fig.5) .

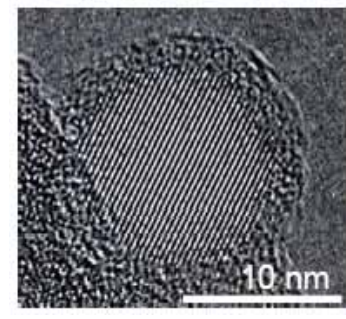

Fig.3 TEM image of a silicon duantum dot

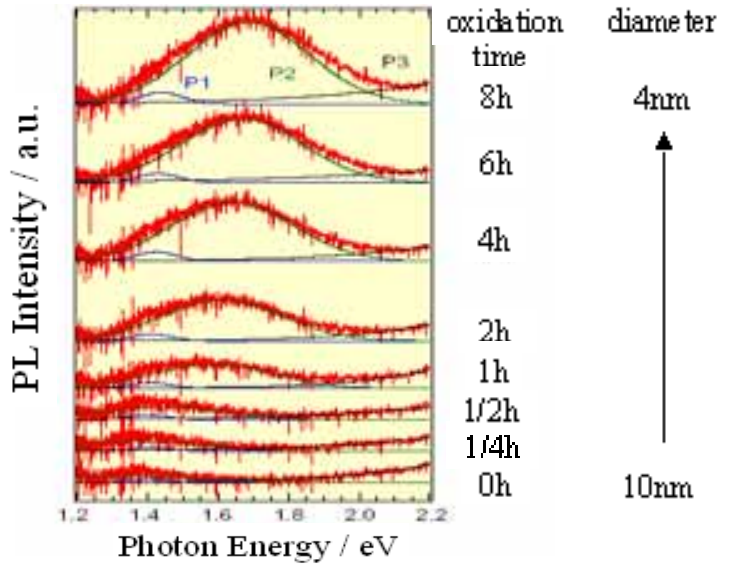

Fig.4 Denendence of the nhotoluminescence snectra on the thermal oxidation time. 


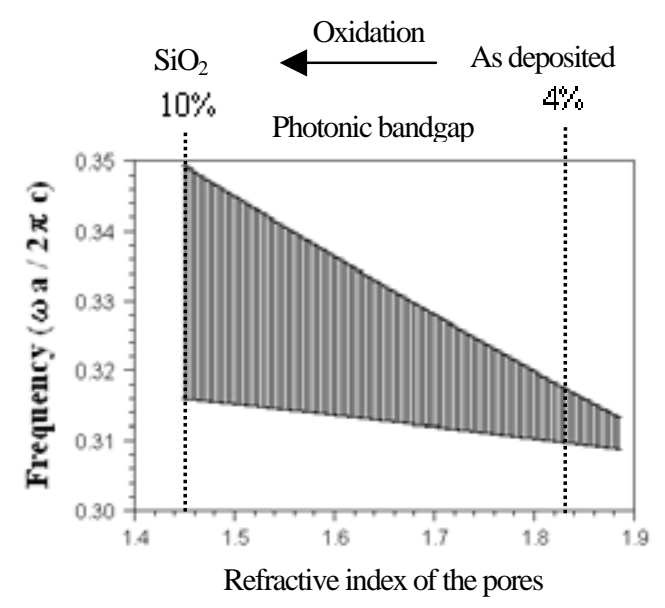

Fig.5 Photonic bandgap dependence on the refractive index of the pores

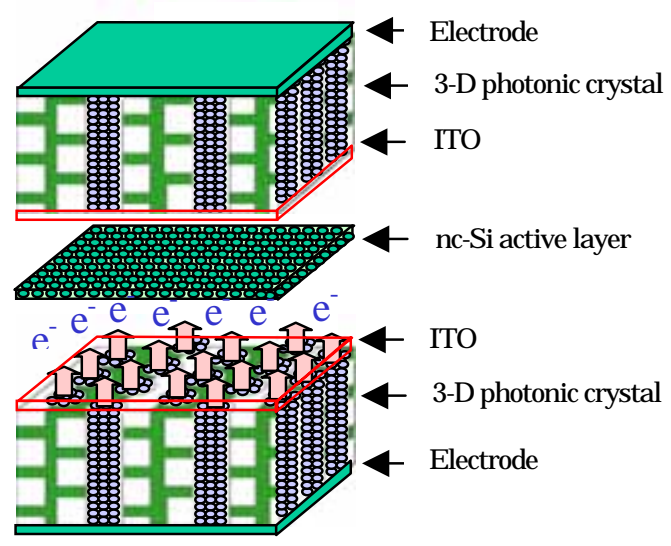

Fig.6 A schematic of the whole device structure

As for the electron emission properties, emission efficiency of about $5 \%$ has already been achieved using the nanocrystalline silicon dots [7], comparable to that for commercially available porous silicon emitters. The superior properties result from the high energy electron emission associated with phonon stop-band formed in the periodic array of nanocrystalline silicon [8]. The advantage of this excitation method is that we can get high energy electron emission under a low voltage to excite the nanocrystalline silicon active layer. This leads to low power consumption favored in the actual implementation in the CMOS integrated circuits. The schematic of the whole device structure is shown in Fig.6.

\section{Conclusion}

Previous studies on nanocrystalline silicon light emitting devices have mainly focused on the control of electronic properties of nanocrystalline silicon in terms of the quantum confinement effect as well as the control of its surface states. As for the control of electromagnetic field, the device structure based on one-dimensional Bragg reflector has been studied most widely to improve the luminescence properties of nanocrystalline silicon. We proposed the ultimate control of electromagnetic field using 3-dimensional photonic crystal structures and showed a preliminary result of its fabrication process. We also proposed an excitation mechanism based on high energy electron emission due to phonon stop-band generated by the periodic array of nanocrystalline silicon, which is different from any conventional excitation methods such as optical pumping and current injection.

Our nanocrystalline silicon optical device features the electron emission structure built utilizing a part of 3-dimensional photonic crystal as a component to build nanocrystalline silicon arrays. Nanocrystalline silicon was fabricated with virtually the same diameter of about $10 \mathrm{~nm}$ using pulsed gas plsma CVD method, and the visible photoluminesece was observed for the oxidized nanocrystalline silicon indicating the quantum confinement effect. Another key feature of our device is that the dimension of nanocrystalline silicon can be controlled simply by changing the thermal oxidation time [9]. This is vital to control the electron emission properties, the luminescence wavelength of nanocrystalline silicon, the refractive index of the structured nanocrystalline silicon arrays, and therefore to optimize the photonic bandgap properties of the whole device.

\section{References}

[1] L. Pavesi, L. Dal Negro, C. Mazzoleni, G. Franzo \& F. Priolo, Nature 408, 440 (2000)

[2] M. J. Chen, J. L. Yen, J. Y. Li, J. F. Chang, S. C. Tsai, and C. S. Tsai, Appl. Phys. Lett. 84, 2163 (2004)

[3] Boyraz O. \& Jalali B, Opt. Express, 12. 5269 - 5273 (2004)

[4] H. Rong, R. Jones, A. Liu, O. Cohen, D. Hak, A. Fang and M. Paniccia, Nature 433, 1 (2005)

[5] S. Uno, K. Nakazato, S. Yamaguchi, A. Kojima, N. Koshida, and H. Mizuta, IEEE Transactions on Nanotechnology, Vol. 2, No. 4, 301 (2003)

[6] T. Ifuku, M. Otobe, A. Itoh and S. Oda, Jpn.. J. Appl. Phys. 36 (1997) 4031-4034

[7] K. Nishiguchi, X. Zhao, and S. Oda, J. Appl. Phys., Vol. 92, No. 5, 2748 (2002)

[8] S.Uno, N. Mori, K. Nakazato, N. Koshida, H. Mizuta, in press for Phys. Rev. B (2005)

[9] J. Omachi, R. Nakamura, K. Nishiguchi, and S. Oda, Mat. Res. Soc. Symp. Proc. Vol. 638, F5. 3.1 (2001) 